\title{
Design and construction of linear laser encoders that possess high tolerance of mechanical runout
}

\author{
Chih-Kung Lee, Chyan-Chyi Wu, Shih-Jui Chen, Liang-Bin Yu, You-Chia Chang, \\ Yeong-Feng Wang, Jau-Yu Chen, and Jeremy Wen-Jong Wu
}

\begin{abstract}
A linearly diffracted laser encoder that has high tolerance of head-to-scale misalignment and a high signal-to-noise ratio is described. The preservation of parallelism between the incident and the diffracted beams, which can be attributed to a built-in folded $1 \times$ telescope, allows for the high alignment tolerance. It can be shown that, by coupling this newly developed circular polarization interferometer configuration with grating scale geometry optimization, one can eliminate the problems associated with signal distortion that arise from various efficiencies of the $p$ - and the $s$-polarized light beams and obtain a high signal-to-noise ratio. Both theoretical and experimental results are presented to confirm the improved results and performance. (C) 2004 Optical Society of America

OCIS codes: $\quad 220.4830,120.4570,120.3180,120.3930,050.1950,120.3940$.
\end{abstract}

\section{Introduction}

Displacement measurements of high sensitivity and accuracy are indispensable to precision machining and nanotechnology. For submicrometer positioning, noncontact measurements are important because even a small load tends to cause deformation and to lead to measurement errors. Of all the noncontact metrology methods developed for precision displacement measurement, the interferometer enjoys the widest popularity. Nevertheless, interferometers suffer from high sensitivity to environmental disturbance, as minute temperature or pressure variations can lead to large measurement errors. ${ }^{1,2}$

In general, optical encoders derive their metrology signals by converting light beam intensity or wavelength to modulations of grating scales. Geometrical optical encoders utilize moire or projections of gratings to obtain the displacement signal from light

C.-K. Lee (cklee@mems.iam.ntu.edu.tw), S.-J. Chen, L.-B. Yu, Y.-C. Chang, Y.-F. Wang, and J.-Y. Chen are with the Institute of Applied Mechanics, National Taiwan University, Taipei 106, Taiwan. C.-K. Lee is also with the Department of Engineering Science and Ocean Engineering, National Taiwan University, which is also the affiliation for J.W.-J. Wu. C.-C. Wu is with the Center for Measurement Standards, Industrial Technology Research Institute, Hsinchu 300, Taiwan.

Received 12 January 2004; revised manuscript received 4 June 2004; accepted 20 July 2004.

0003-6935/04/315754-09\$15.00/0

(C) 2004 Optical Society of America intensity modulations; in this approach the grating pitch is limited to a $10-\mu \mathrm{m}$ range because of the diffraction limit that in turn limits the best resolution to $100 \mathrm{~nm}$ or so. A diffractive laser encoder, however, not only can circumvent the diffraction limitation but can also encode the displacement signal by taking advantage of the diffraction signal. The phase encoded within the diffractive light beam when the grating scale moves provides the diffractive laser encoder with a way to achieve nanometer measurement accuracy. Interfering diffractive light beams with a phase modulated by the grating scale lead to a grating interferometer that transforms the underlying measurement scale from a laser wavelength type to a grating pitch type. As the displacement does not depend explicitly on the optical path difference, it enjoys a higher degree of immunity to environmental disturbances. Such a property is extremely valuable for many applications and is one of the main differences that separate interferometers from diffractive laser encoders.

With the potential to achieve better than a few nanometers' metrology accuracy in vastly different environments, significant research and development efforts have been expended on this issue and have resulted in many innovative designs. ${ }^{1-5}$ It has been found that wave-front aberrations induced by runout of the grating-scale movement cause the largest measurement error for laser encoders. The only design known to deal with this problem directly is that of a laser encoder that was originally developed for use with magnetic disk drive servo writing tasks. ${ }^{6}$ In 


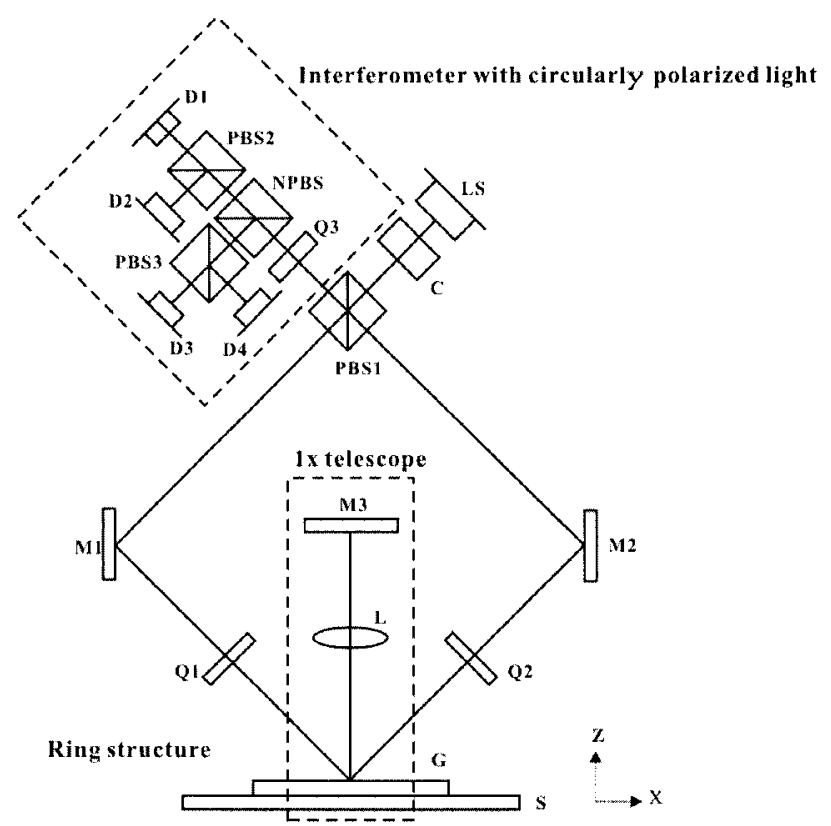

Fig. 1. Configuration of the newly developed laser encoder system, DiLENS. The grating moves in the $x$ direction.

this design, two folded $1 \times$ telescopes are used to compensate automatically for grating wave-front aberrations. However, this design still suffers from the difference experienced by the two distinct folded $1 \times$ telescopes as well as from the phase decoding errors induced by the differences in grating diffraction efficiency between the $p$ - and the $s$-polarized light beams, which appear as quadrature signal distortions.

In another laser encoder design ${ }^{7}$ the diffractive laser encoder removes the grating-scale-induced wave-front aberration by developing a 1/4-pitch gradient-index (GRIN) lens with a reflective coating only at the center of its back side. A spatial filter is formed by integration of the GRIN lens and the back side's central coating, which in effect removes all high-order wave-front aberrations. However, this so-called cat's-eye approach trades off light beam energy for wave-front cleanness, leading to inefficient use of light that limits the achievable maximum signal-to-noise ratio.

In our new design the diffractive laser encoder configuration possesses a high tolerance of high-speed mechanical runout while at the same time it maintains an efficient use of light to arrive at a high signal-to-noise ratio. Our design principle, the optical configuration that we developed, and the experimental data are detailed in this paper. In addition, the optimization criteria for achieving a high signalto-noise ratio are presented as well.

\section{Optical Configuration Design}

Our newly proposed diffractive laser encoder system (DiLENS) is shown in Fig. 1. The optical system is composed of three subsystems, a ring structure, a $1 \times$ telescope, and a circular polarization interferometer, each of which has a distinct function. The major function of the ring structure is to guide the laser beam generated by the laser source to the linear grating scale and to the telescope. It then guides the +1 st- and -1 st-order diffracted light to the circular polarization interferometer subsystem. The laser source (LS) used in the DiLENS is a diode laser with a 780-nm wavelength, and it is oriented such that the emitted laser beam is linearly polarized at $45^{\circ}$, which in turn equalizes the intensities of the $p$ - and $s$-polarized light beams after polarizing beam splitter PBS1 (Fig. 1). A collimator (C) is aligned in front of the laser source to collimate the laser beam. The $p$-polarized light beam transmitting through PBS1 forms the left arm of the ring structure. The right arm is formed as a result of the reflected $s$-polarized light beam. Before reaching linear grating $\mathrm{G}$, the $p$-polarized beam is reflected by mirror $\mathrm{M} 1$ and converted into circularly polarized light after passing through quarter-wave plate Q1. The grating pitch and the mirror orientation are set to the -1 st-order diffracted light perpendicular to the grating surface. The -1 st-order diffracted light is then guided perpendicularly back onto the grating by the $1 \times$ telescope section for diffraction. After being diffracted twice at the -1 st order, the beam propagates back along the same route but in a direction opposite that of the original incident light beam. This returning beam passes through Q1 again and is then converted to $s$-polarized light. PBS1 then reflects this beam to the circular polarization interferometer subsystem. Similarly, the $s$-polarized light beam after PBS1 is guided to the right-hand arm of the ring structure and is twice diffracted by the grating scale at the 1st order, where it then travels back to the circular polarization section and becomes $p$ polarized. By diffraction twice, at the +1 st and the -1 st orders, the displacement signal is embedded into the phases of the two returning light beams and forms the two interference arms of the circular polarization interferometer section. The phase modulation exerted by the grating scale can be interpreted as a Doppler frequency shift caused by the grating-scale velocity. Doppler frequency shifts $\Delta w_{+1}$ and $\Delta w_{-1}$ relate to grating-scale velocity $u_{x}$ as follows:

$$
\begin{aligned}
& \Delta w_{+1}=2 \Delta w=u_{x} \times \frac{4 \pi}{\lambda} \times \sin \theta, \\
& \Delta w_{-1}=-2 \Delta w=-u_{x} \times \frac{4 \pi}{\lambda} \times \sin \theta,
\end{aligned}
$$

where $\lambda$ represents the wavelength of the light source and $\theta$ is the 1st-order diffraction angle. As a phase signal can be obtained by integration of the frequency signal, displacement can be retrieved from the diffractive laser encoder. Detailed theoretical results are presented here.

\section{A. Four-Photodiode Circular Polarization Interferometer}

Directional ambiguity, the situation in which the interferometer cannot discern the direction of move- 


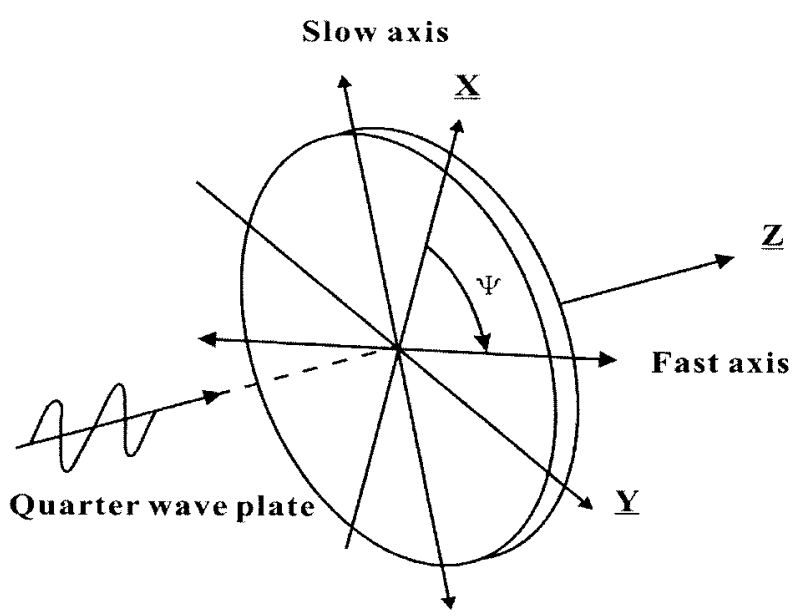

Fig. 2. Definition of fast-axis azimuth angle for Q1 and Q2. The positive $Z$ axis starts from mirror M1 to grating $\mathrm{G}$ for $\mathrm{Q} 1$ and from M2 to grating G for Q2.

ment by analyzing the signal, is an important issue when one is choosing an interferometer as a metrology tool. ${ }^{8}$ Generally, a frequency-shifting device such as an acoustic-optical modulator is used to modulate the signal to permit the direction of movement to be discerned. ${ }^{9}$ By comparison, a circular polarization interferometer adopts quadrature signals to deal with this ambiguity. In an attempt to minimize the overall system volume, we incorporated a circular polarization interferometer into the DiLENS. A four-photodiode configuration was adopted to remove the dc part of the quadrature signal while the ac component was retained to decode the displacement signal.

Then we applied Jones calculus ${ }^{10}$ to derive the relationship between displacement and phase. The electrical fields of the light beams that have been diffracted twice, at the -1 st and the +1 st orders, are

$$
\begin{aligned}
& V_{1}=\left\{\begin{array}{c}
-k_{p}(\theta) \times k_{p}(0) i \\
k_{s}(\theta) \times k_{s}(0)
\end{array}\right\} \exp [i(\omega-2 \Delta \omega) t], \\
& V_{2}=\left\{\begin{array}{c}
k_{p}(\theta) \times k_{p}(0) \\
k_{s}(\theta) \times k_{s}(0) i
\end{array}\right\} \exp [i(\omega+2 \Delta \omega) t],
\end{aligned}
$$

where subscripts 1 and 2 represent the left and the right arms and $k_{p}(\theta)$ and $k_{s}(\theta)$ denote the diffraction efficiencies of the $p$ - and the $s$-polarized light, respectively, when the incident angle is $\theta$, and $i$ denotes $(-1)^{1 / 2}$. It is worth noting that orientation angles $\psi$ of quarter-wave plates Q1 and Q2 (Fig. 1) can be set at $+45^{\circ}$ and $-45^{\circ}$, respectively (Fig. 2). After the light beams pass through Q1 and Q2, their electrical fields immediately before they return to PBS1 become

$$
\begin{aligned}
U_{1} & =\left\{\begin{array}{c}
-b i \\
a
\end{array}\right\} \exp [i(\omega-2 \Delta \omega) t], \\
U_{2} & =\left\{\begin{array}{c}
a \\
-b i
\end{array}\right\} \exp [i(\omega+2 \Delta \omega) t],
\end{aligned}
$$

where

$$
\begin{gathered}
a=2^{-1 / 2} \times\left[k_{p}(\theta) \times k_{p}(0)+k_{s}(\theta) \times k_{s}(0)\right], \\
b=2^{-1 / 2} \times\left[k_{p}(\theta) \times k_{p}(0)-k_{s}(\theta) \times k_{s}(0)\right] .
\end{gathered}
$$

After the light passes through PBS1, the proper polarization components of $U_{1}$ and $U_{2}$ that enter into the detection subsystem become

$$
\begin{aligned}
U_{1}{ }^{\prime} & =\left\{\begin{array}{l}
0 \\
a
\end{array}\right\} \exp [i(\omega-2 \Delta \omega) t], \\
U_{2}{ }^{\prime} & =\left\{\begin{array}{l}
a \\
0
\end{array}\right\} \exp [i(\omega+2 \Delta \omega) t] .
\end{aligned}
$$

Examining Eqs. (8)-(10), we can conclude that the DiLENS does not suffer from any diffraction efficiencies differences $b$ [Eq. (8)] of grating scale with respect to the different polarization states of the incident light beams because $b$ does not appear in the final signal. After these two light beams pass through quarter-wave plate Q3 (Fig. 1), their polarization states they are converted into left and right circularly polarized light beams:

$$
\begin{aligned}
& W_{1}=2^{-1 / 2} a\left[\begin{array}{l}
i \\
1
\end{array}\right] \exp [i(\omega-2 \Delta \omega) t], \\
& W_{2}=2^{-1 / 2} a\left[\begin{array}{l}
1 \\
i
\end{array}\right] \exp [i(\omega+2 \Delta \omega) t],
\end{aligned}
$$

where $\Delta \omega$ is the Doppler frequency shift induced by the grating velocity as shown in Eqs. (1) and Eq. (2).

Nonpolarized beam splitter NPBS2 (Fig. 1) splits combined light beams $W_{1}$ and $W_{2}$, which are then further split by polarized beam splitter PBS2. Both the transmitted and the reflected beams split by the PBS2 are detected by the photodiodes. Similarly, the beam reflected by NPBS2 is split by polarized beam splitter PBS3 and then captured by the photodiodes. The quadrature signal is generated by PBS3 oriented at $45^{\circ}$ spatially with respect to that of PBS2. The intensities detected by the four photodiodes can be derived as

$$
\begin{aligned}
& I_{D 1^{\prime}}=2 a^{2}+2 a^{2} \times \cos (4 \Delta \omega \times t), \\
& I_{D 2}{ }^{\prime}=2 a^{2}-2 a^{2} \times \cos (4 \Delta \omega \times t), \\
& I_{D 3^{\prime}}=2 a^{2}+2 a^{2} \times \sin (4 \Delta \omega \times t), \\
& I_{D 4}{ }^{\prime}=2 a^{2}-2 a^{2} \times \sin (4 \Delta \omega \times t) .
\end{aligned}
$$

The dc term is removed by the differential amplification of photodiodes D1 and D2, as are the signals from photodetectors D3 and D4. The ac components from D1 to D4 are the quadrature signals needed to form a Lissajous circle pattern:

$$
\begin{aligned}
& I_{P}=4 a^{2} \times \cos (4 \Delta \omega \times t), \\
& I_{Q}=4 a^{2} \times \sin (4 \Delta \omega \times t) .
\end{aligned}
$$


Table 1. Destination of Light Beams Associated with Several Orientations of Quarter-Wave Plates Q1 and Q2 in Fig. 1

\begin{tabular}{|c|c|c|c|c|c|c|c|c|}
\hline \multirow[b]{2}{*}{ Polarization } & \multicolumn{8}{|c|}{ Type of Beam } \\
\hline & \multicolumn{4}{|c|}{$p$-Polarized } & \multicolumn{4}{|c|}{$s$-Polarized } \\
\hline Optical path & & & & & & & & f \\
\hline $\begin{array}{l}\text { Diffraction orders } \\
\text { Orientation }^{a}\end{array}$ & Twice 1st & Oth & -1 st and +1 st & -2 nd & Twice 1st & Oth & +1 st and -1 st & 2nd \\
\hline$\left(+45^{\circ},+45^{\circ}\right)$ & $\mathrm{PD}^{b}$ & Laser & Laser & $\mathrm{PD}$ & $\mathrm{PD}$ & Laser & Laser & $\mathrm{PD}$ \\
\hline$\left(+45^{\circ},-45^{\circ}\right)$ & $\mathrm{PD}$ & $\mathrm{PD}$ & $\mathrm{PD}$ & PD & PD & $\mathrm{PD}$ & $\mathrm{PD}$ & PD \\
\hline$\left(-45^{\circ},+45^{\circ}\right)$ & $\mathrm{PD}$ & $\mathrm{PD}$ & PD & $\mathrm{PD}$ & $\mathrm{PD}$ & $\mathrm{PD}$ & $\mathrm{PD}$ & $\mathrm{PD}$ \\
\hline$\left(-45^{\circ},-45^{\circ}\right)$ & $\mathrm{PD}$ & Laser & Laser & $\mathrm{PD}$ & $\mathrm{PD}$ & Laser & Laser & $\mathrm{PD}$ \\
\hline
\end{tabular}

a The quarter-wave plates' fast-axis azimuth angles (Fig. 2) are expressed as $\left(\psi_{\mathrm{Q} 1}\right.$ and $\left.\psi_{\mathrm{Q} 2}\right)$.

${ }^{b} \mathrm{PD}$, Photodiode.

By means of phase decoding techniques, 6,11 the phase of the quadrature signals $\Phi$ and the displacement of grating $\Delta X$ are related as follows:

$$
\begin{aligned}
\Phi & =\int \mathrm{d} \Phi \\
& =\int 4 \Delta \omega \mathrm{d} t \\
& =\int \frac{8 \pi}{\lambda} u_{x} \sin \theta \mathrm{d} t \\
& =\frac{8 \pi}{\lambda} \Delta X \sin \theta .
\end{aligned}
$$

After applying the grating equations, we can express the diffraction angle of the 1st order as follows:

$$
\sin \theta=\lambda / d,
$$

where $d$ represents the pitch of the grating.

Substituting Eq. (20) into Eq. (19) leads to

$$
\Delta X=\frac{\Phi \times d}{8 \pi} .
$$

Equation (21) shows that the phase of the Lissajous circle changes by $8 \pi$ whenever the grating moves by a pitch. Equation (21) clearly demonstrates that the reference scale of a diffractive laser encoder is in grating-scale pitch format, which is different from the laser source wavelengths used in traditional interferometers. As a grating-scale pitch tends to be more environmentally stable than that of air, a diffractive laser encoder is typically more robust than traditional interferometers.

Simple analysis of the proper propagation of the \pm 1 st-order diffracted light indicates that the orientation of Q1 and Q2 influences how the 0th- and the 2nd-order diffracted beams propagate to the photodetectors or back to the laser. To prevent laser mode hopping we guide the 0th-order diffracted light away from the laser cavity, as it has the strongest light intensity. The destinations of the light beams with different diffraction orders and different Q1 and Q2 orientations are summarized in Table 1. It is clear from Table 1 that $\left(+45^{\circ},-45^{\circ}\right)$ and $\left(-45^{\circ},+45^{\circ}\right)$ quarter-wave plate orientations guided to the 0th order diffract beams away from the laser cavity. Instead, the 0th-order diffracted beams are sent to the photodiodes. We can see that the 0th-order diffracted light beam will not decrease the quadrature signals in this four-photodiode configuration as differential amplifications are adopted.

In addition to the 0th-order diffracted beams, 2ndorder diffracted light, which possesses the same Doppler frequency shift as that of the twice diffracted 1storder light beam, also enters the photodiodes. A moire pattern between the two light beams mentioned above may appear at misaligned positions. Either of two approaches can be adopted to solve this problem. One is to pursue precise alignment and the other is to develop a slightly modified configuration. This configuration inserts a quarter-wave plate into the $1 \times$ telescope (instead of using two quarter-wave plates, Q1 and Q2) in the configuration (Fig. 1), which sends the 2nd-order diffracted light back to the laser cavity where the moire noise can be completely eliminated. This alternative configuration also has the advantage of reduced size, as fewer optical components are used. The destinations of the unwanted light beams in this configuration are given in Table 2 , which indicates that the light returning after the +1 st and the -1 st orders, as well as the 2nd-order diffraction light beam, goes back to the laser cavity. No laser mode hopping is predicted, as the intensities of the light beams are low because of the low associated diffraction efficiency, as we verified experimentally.

\section{B. Folded $1 \times$ Telescope}

The primary alignment tolerance of the DiLENS of mechanical runout can actually be attributed to the efficiency of a built-in folded $1 \times$ telescope design consisting of lens L and mirror M3 (Fig. 1). The distances between the lens and the grating as well as between the lens and the mirror are set to be equal to the focal length of lens L. We use paraxial analysis $^{12,13}$ herein to examine how the $1 \times$ telescope optics can improve the system's tolerance. Note that the tilt angle of the grating during operation is typically 


\begin{tabular}{|c|c|c|c|c|c|c|c|c|}
\hline \multirow[b]{2}{*}{ Polarization } & \multicolumn{8}{|c|}{ Type of Beam ${ }^{b}$} \\
\hline & \multicolumn{4}{|c|}{$p$-Polarized } & \multicolumn{4}{|c|}{$s$-Polarized } \\
\hline Optical path & & & & & & & & \\
\hline $\begin{array}{l}\text { Diffraction order } \\
\text { Orientation }^{a}\end{array}$ & Twice 1st & Oth & -1 st and +1 st & $-2 \mathrm{nd}$ & Twice +1 st & Oth & -1 st and +1 st & $2 \mathrm{nd}$ \\
\hline$\psi=+45^{\circ}$ & $\mathrm{PD}^{b}$ & $\mathrm{PD}$ & Laser & Laser & $\mathrm{PD}$ & $\mathrm{PD}$ & Laser & Laser \\
\hline$\psi=-45^{\circ}$ & $\mathrm{PD}$ & $\mathrm{PD}$ & Laser & Laser & $\mathrm{PD}$ & $\mathrm{PD}$ & Laser & Laser \\
\hline
\end{tabular}

${ }^{a}$ Fig. 2.

${ }^{b} \mathrm{PD}$, photodiode.

less than $1^{\circ}$, and a properly designed lens can certainly justify the paraxial analysis used. Consider the optical layout shown in Fig. 3, where $h_{0}$ and $\eta_{0}$ represent the height and the emitted angle, respectively, of the light beam diffracted from the grating. If the grating is perfectly aligned, height $h_{0}$ and angle $\eta_{0}$ will both be zero. $\Delta Z$ represents the stand-off of the grating caused by the mechanical runout during operation, and $f$ is the focal length of the lens. The height and the angle of light beam coming from the folded $1 \times$ telescope can be expressed as ${ }^{12}$

$$
\begin{gathered}
{\left[\begin{array}{ccc}
1 & -f & -\Delta Z \\
0 & 1 & Z
\end{array}\right]\left[\begin{array}{cc}
1 & 0 \\
1 / f & 1
\end{array}\right]\left[\begin{array}{cc}
1 & -f \\
0 & 1
\end{array}\right]\left[\begin{array}{cc}
1 & 0 \\
0 & -1
\end{array}\right]\left[\begin{array}{ll}
1 & f \\
0 & 1
\end{array}\right]} \\
{\left[\begin{array}{cc}
1 & 0 \\
-1 / f & 1
\end{array}\right]\left[\begin{array}{cc}
1 & f+\Delta Z \\
0 & 1
\end{array}\right]\left\{\begin{array}{l}
h_{0} \\
\eta_{0}
\end{array}\right\}} \\
=\left\{\begin{array}{c}
-h_{0}-2 \Delta Z \times \eta_{0} \\
\eta_{0}
\end{array}\right\} .
\end{gathered}
$$

Equation (22) clearly denotes that the angle of the returning beam is identical to the incident angle for all stand-off and tilt. This means that the returning light beam always propagates in exactly the opposite direction from the original incident light beam. It basically states that a folded $1 \times$ telescope resembles a corner cube with its tip located at the mirror's reflection surface such as to guarantee parallelism between the incident and the returning light beams. Such a property prevents deviation of the light beam

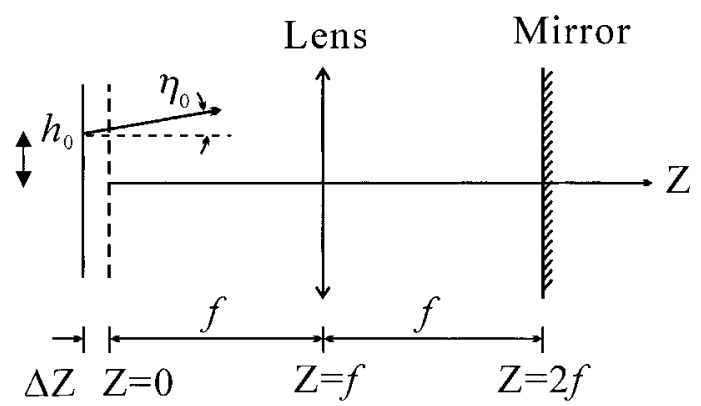

Fig. 3. Folded $1 \times$ telescope configuration with $h_{0}$ and $\eta_{0}$, which represent the height and the emitting angle, respectively, of the light beam diffracted from the grating. when runout is present and significantly enhances the alignment tolerance.

In spite of some apparent similarities between the folded $1 \times$ telescope and the so-called cat's-eye configuration, ${ }^{7}$ there are fundamental differences that enable the DiLENS to have superior performance. This case is an excellent example: A single component change can lead to a dramatic performance difference in a laser encoder system. Under a paraxial assumption, the 1/4-pitch GRIN lens ${ }^{14}$ in a cat's-eye configuration is equivalent to a typical thin lens set at the middle position of a rod whose focal length equals half of the rod's length. The cat's-eye configuration cannot be designed as a $1 \times$ telescope because the GRIN lens would then come into contact with the grating. As the distance between the grating and the lens cannot be set to $f$, the analysis summarized by Eq. (22) shows that the deviation in height is caused by the oblique incidence.

In the cat's-eye configuration, the spatial filter design trades off wave-front cleanness for light energy, which can lead to degradation in the signal-to-noise ratio. The newly disclosed configuration, however, combines the conjugate wave created by the folded $1 \times$ telescope and the grating symmetry to eliminate aberrations associated with grating-scale runout without incurring any energy loss. More specifically, because the distance between the grating and the lens as well as the distance between the lens and the mirror equals the focal length of the lens, a simple relationship between the incident wave and the returned wave exists and can be shown by use of Fourier optics, where ${ }^{15}$

$$
u_{r}(x, y)=u_{i}(-x,-y)
$$

and where $u_{i}$ represents the incident light beam emitted from the grating and $u_{r}$ is the light beam returning to the grating. The inverse symmetry shown in Eq. (23) has its origin rooted in the two Fourier transforms when the light beam passes through the lens twice. Because the aberration introduced by a linear grating is still a plane wave, incident wave $u_{i}$ has the form

$$
u_{i}(x, y)=A \exp \left[j\left(k_{x} x+k_{y} y\right)\right] .
$$

Here $A$ represents the amplitude of the wave, and the phase term $\exp \left[j\left(k_{x} x+k_{y} y\right)\right]$ shows the phase error caused by grating-runout-induced aberrations. As a 


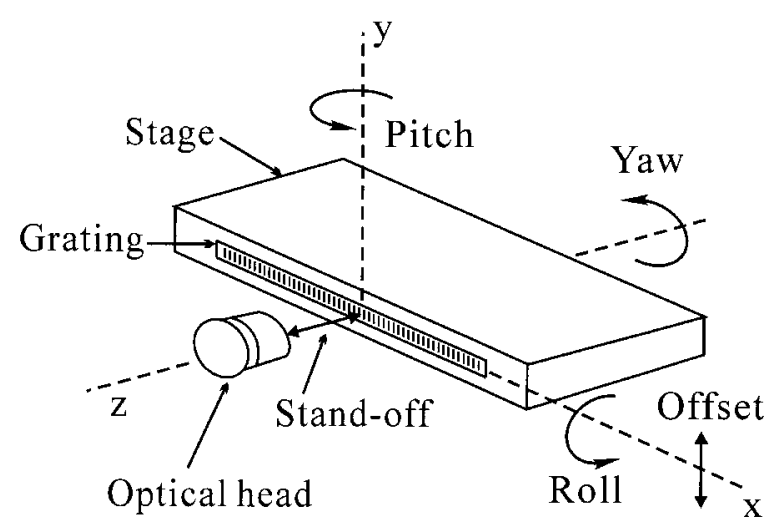

Fig. 4. Definition of runout for a moving grating scale.

result of this inverse symmetry, the returning wave will become

$$
u_{r}(x, y)=A \exp \left[-j\left(k_{x} x+k_{y} y\right)\right],
$$

which is conjugate to the incident light beam. The phase error is canceled out when the returning light beam impinges upon the grating again. In other words, the wave-front reconstruction concept of a folded $1 \times$ telescope really is an optical phaseconjugation technique that has actions similar to those of a four-wave mixing technique when one is dealing with plane-wave aberration.

To further verify the alignment tolerance of the DiLENS, optical ray tracing was adopted to enable us to pursue further simulations. ${ }^{16}$ Two misaligned conditions were found to be most detrimental. The first condition causes the returning light beam to separate, which in turn significantly reduces the ac portion of the interference signal while the dc portion is retained. As the modulated signal is embedded within the ac portion of the interference signal, this condition reduces the signal strength and the signalto-noise ratio. The second condition moves the light spot out of the photodiode range to reduce the overall interference signal's strength, no matter whether it is ac or dc. Therefore our tolerance analysis was performed under the following two conditions:

1. The separation captured by all the photodiodes between the two beams was less than one fourth of the diameter of the beam, which is $2 \mathrm{~mm}$ in the actual DiLENS setup.

2. The center of the beam spots was within the photodiodes' range, which is $4 \mathrm{~mm}$ in the actual DiLENS setup.

The mechanical runout associated with the grating scale can be as defined in Fig. 4, which includes roll, pitch, yaw, stand-off, and offset. Using a ray-tracing program ${ }^{16}$ yields the movements of the misaligned light spots shown in Fig. 5. It is clear that yaw and offset do not influence the spot position; i.e., the DiLENS is immune from the effects of yaw and the offset. Furthermore, pitch was found to be the primary cause of beam separation, as the positions of the

\begin{tabular}{|c|c|}
\hline Alignment parameter & Position of chief rays $(+,-)$ \\
\hline Roll & \\
\hline Pitch & \\
\hline Yaw & 0 \\
\hline Stand-off & \\
\hline Offset & \\
\hline
\end{tabular}

Fig. 5. Qualitative representation of misaligned beam spots for photodiodes under different runout conditions in which separation was found as a result of pitch only.

two returning light spots move together when the grating scale experiences roll or stand-off runout. The quantitative results derived from the simulation are shown in Fig. 6, and the detailed tolerance of the DiLENS is summarized in Table 3. Comparing the data obtained and the specifications of the state-ofthe-art diffractive laser encoder ${ }^{7}$ shows that the DiLENS has a tolerance of runout that is higher by a factor of 6-20 than those of conventional systems. ${ }^{17}$

\section{Effect of Grating Geometry}

It should be noted that perfect quadrature signals result in a circular pattern in Lissajous space. However, a grating generally has different diffraction efficiencies for $p$-polarized and $s$-polarized incident light beams, and this effect tends to distort the circular Lissajous pattern, causing it to become elliptical, which in turn induces errors during phase decoding. ${ }^{18,19}$ The DiLENS is immune to this effect, and it is clear from Eqs. (17) and (18) that $a$ must be maximized to enhance the interference signal and thus to improve the grating's diffraction efficiency. It is known that grating profile, pitch, duty cycle, and depth will all influence diffraction efficiency. From Eq. (7) it is clear that maximizing a maximizes $\left[k_{p}(\theta) k_{p}(0)+k_{s}(\theta) k_{s}(0)\right]^{2}$.

A rigorous coupled-wave analysis (RCWA) has been developed over the years to serve as an exact formulation for the grating diffraction problem that specifies the grating's responses to various polarizations, especially when the grating pitch is close to or shorter than the wavelength of light. ${ }^{20-23}$ To optimize the grating-scale design we used a commercially available software package based on RCWA to design the grating geometry to obtain the optimal $\left[k_{p}(\theta) k_{p}(0)\right.$ $\left.+k_{s}(\theta) k_{s}(0)\right]^{2}$. Experimental verification of the simulated results is presented here.

\section{Experimental Setup}

A series of experiments was carried out to verify the validity of the theories described above. The experimental setup, adopted to verify the alignment toler- 

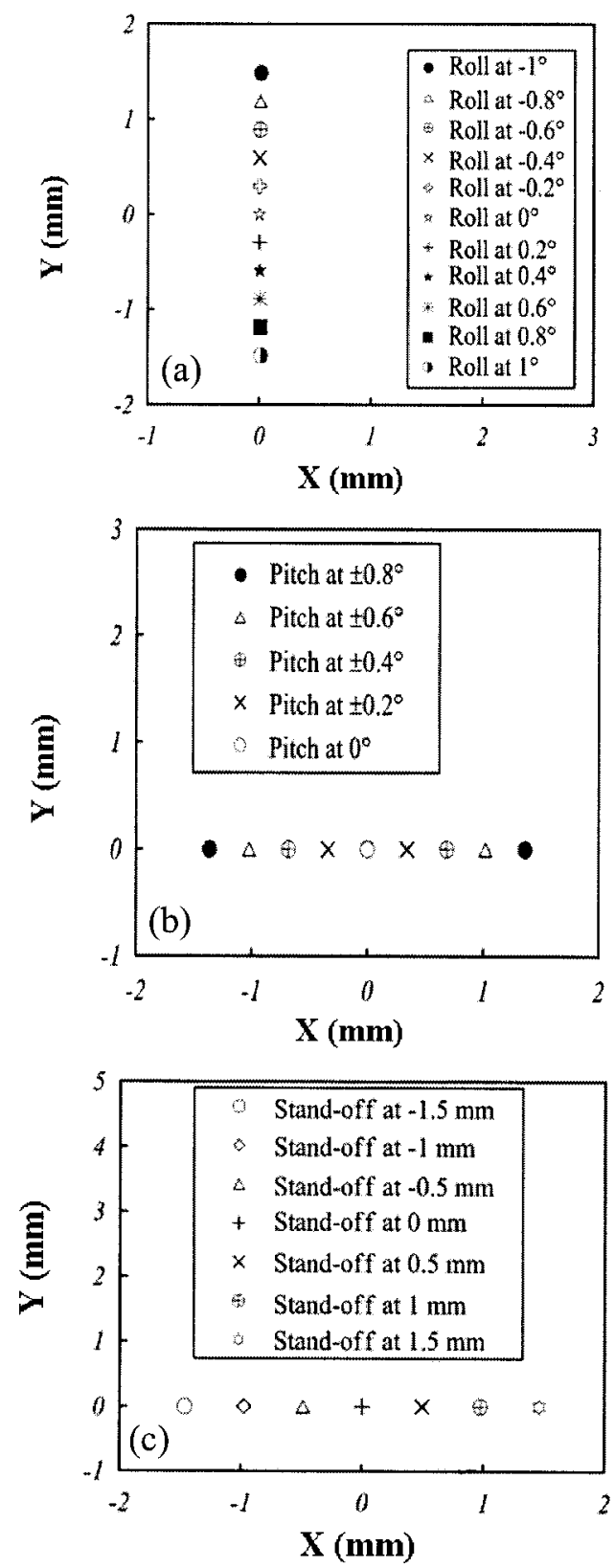

Fig. 6. Spot location of photodiode for roll, pitch, and stand-off runout.

Table 3. Alignment Tolerance of the DiLENS As Determined by Ray Tracing and the Experimental Setup ${ }^{a}$

\begin{tabular}{|c|c|c|c|}
\hline $\begin{array}{c}\text { Alignment } \\
\text { Tolerance }\end{array}$ & $\begin{array}{l}\text { Calculated by } \\
\text { Ray Tracing }{ }^{b}\end{array}$ & $\begin{array}{c}\text { Experimental } \\
\text { Data }\end{array}$ & $\begin{array}{l}\text { State-of-Art } \\
\text { Encoder }^{c}\end{array}$ \\
\hline Roll (arc min) & \pm 60 & \pm 58 & \pm 3 \\
\hline Pitch (arc min) & \pm 20 & \pm 23 & \pm 20 \\
\hline Yaw (deg) & \pm 3 & \pm 3.2 & \pm 20 \\
\hline Stand-off (mm) & \pm 1.5 & \pm 1.3 & \pm 0.2 \\
\hline Offset $(\mathrm{mm})$ & \pm 2 & \pm 2 & \pm 0.3 \\
\hline
\end{tabular}

${ }^{a}$ The tolerance specification of the state-of-art encoder is quoted from Ref. 17 for comparison.

${ }^{b}$ Ref. 16 .

${ }^{c}$ Ref. 7.

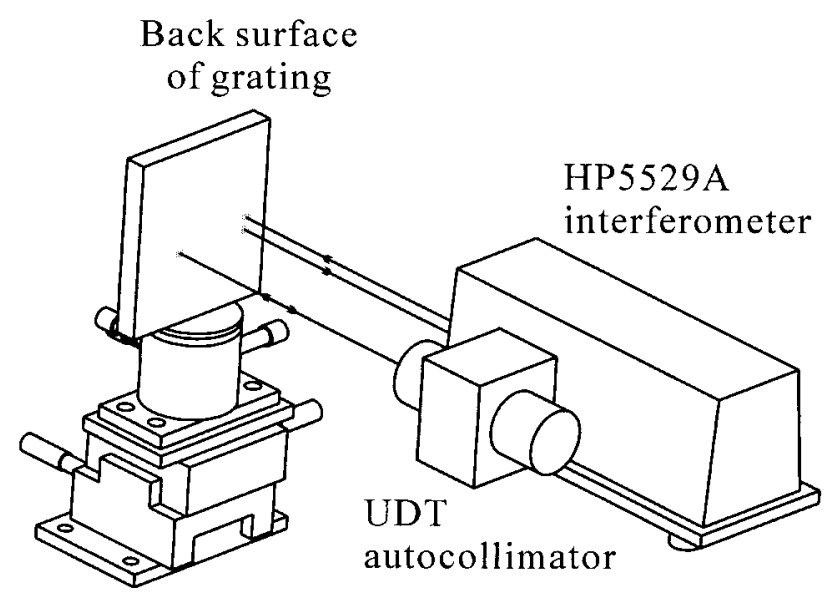

Fig. 7. Experimental setup for measuring grating-scale runout and determining the alignment tolerance.

ance as shown in Fig. 7, was developed based on an autocollimator ${ }^{24}$ and a two-frequency interferometer system. ${ }^{25}$ The autocollimator measures the change in grating angle, and the interferometer determines the additional displacement caused by the rigid body rotation that must be compensated for. The measured alignment tolerance is shown in Table 3 and agrees well with the simulation. In addition, comparisons of the experimental and the simulated dif-
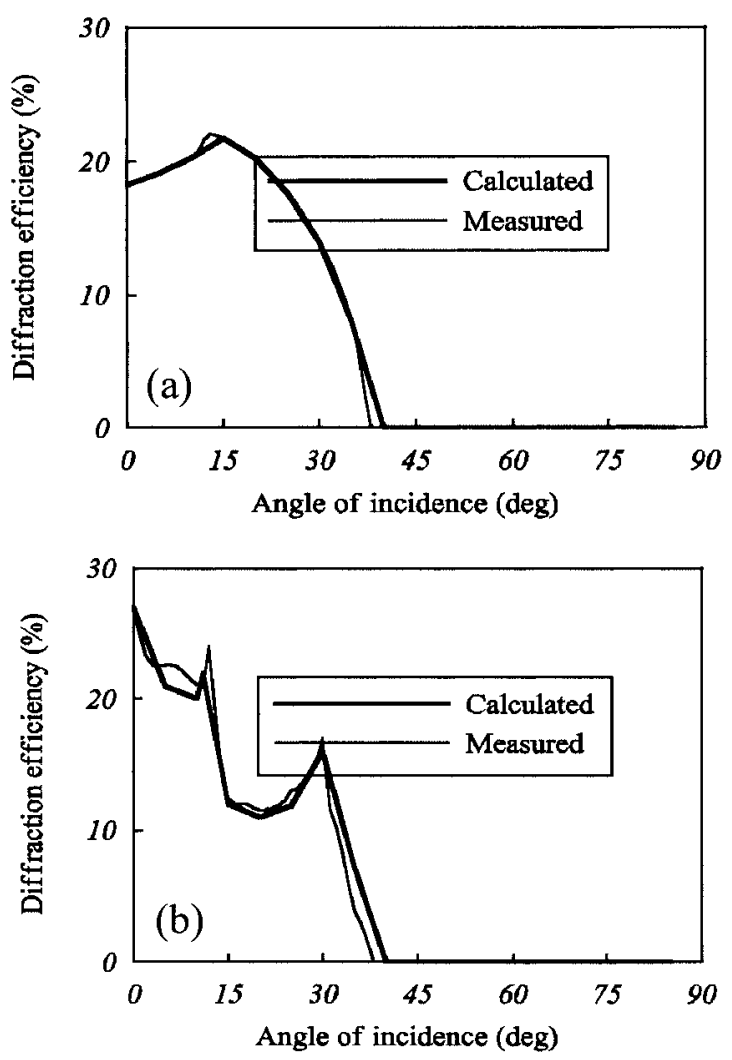

Fig. 8. Diffraction efficiency of a grating scale at different incidence angles, where the simulated data and the experimental data agree well with each other: (a) measured TE efficiencies, (b) measured TM efficiencies. 


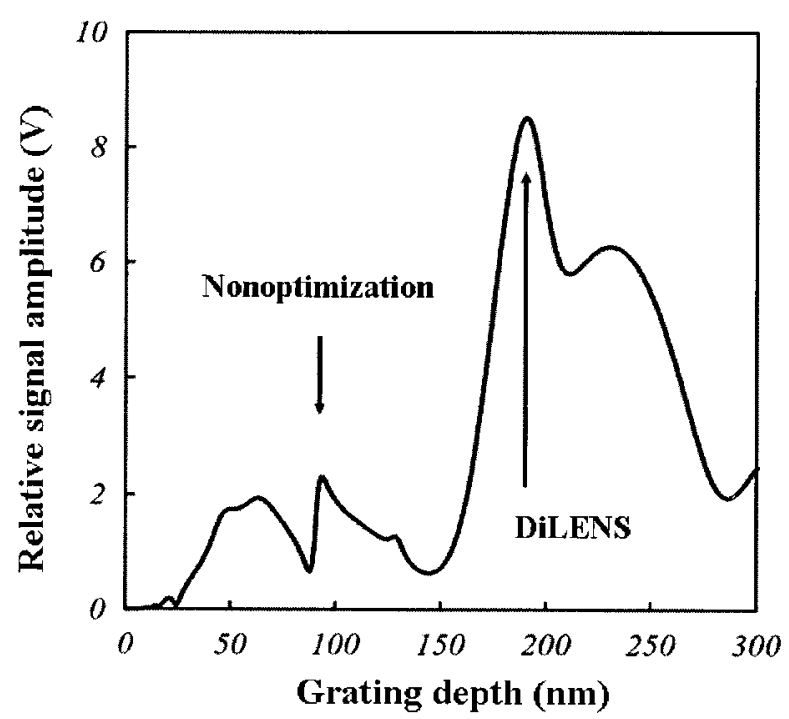

Fig. 9. Simulation of the optimization criterion $\left[k_{p}(\theta) k_{p}(0)+\right.$ $\left.k_{s}(\theta) k_{s}(0)\right]^{2}$ at various grating depths; the optimized depth was 190 $\mathrm{nm}$.

fraction efficiencies at various incident angles are shown in Fig. 8. It is clear from the data shown that simulation results obtained with the RCWA software agree well with the experimental data.

With the validity of the RCWA verified, we performed many more simulations to locate the optimum grating-scale geometry. Finally, a sine profile with a $190-\mathrm{nm}$ grating depth was found to yield the maximum signal-to-noise ratio. This result can be further demonstrated by the data. The simulated diffraction efficiency and its variation relative to grating depth are shown in Figs. 9 and 10, respectively. Compared to the 90-nm grating depth adopted in today's state-of-the-art diffractive laser encoders, ${ }^{7}$

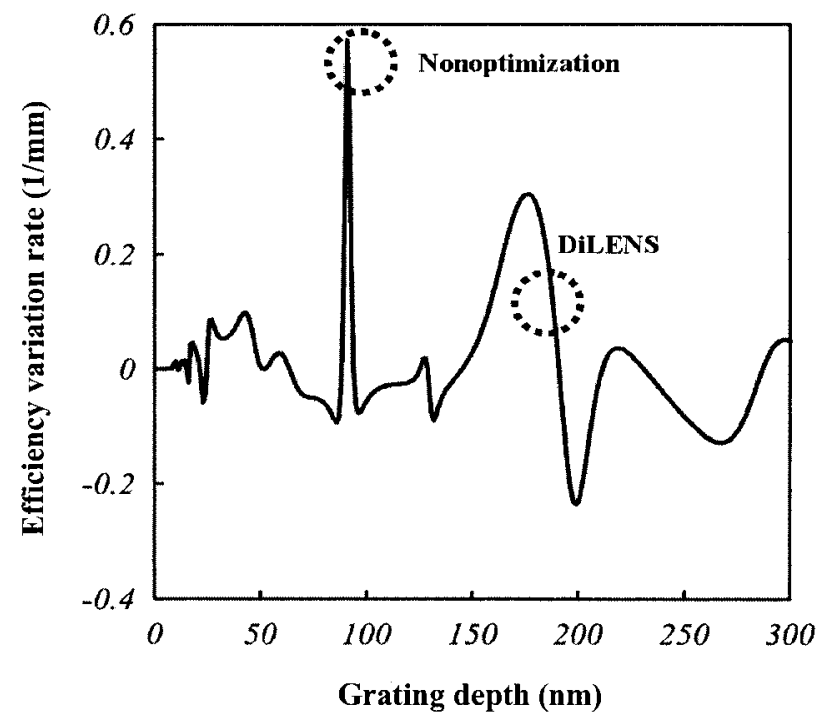

Fig. 10. Simulation of rate of variation in diffraction efficiency versus grating depth. The variation rate was minimum at a 190-nm grating depth.

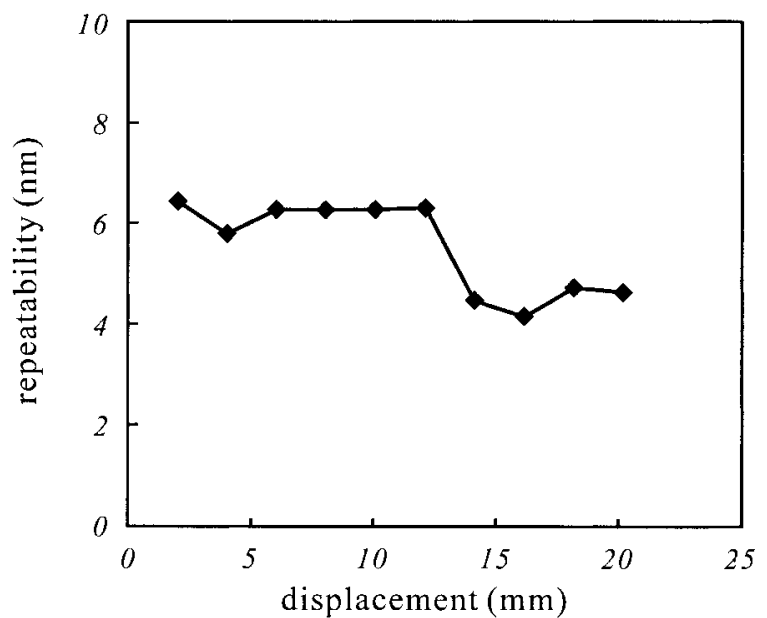

Fig. 11. Experimental results of repeatability tests in which 10 data sets (at 2, 4, 6, 8, 10, 12, 14, 16, 18, and $20 \mathrm{~mm}$ ) were obtained. The repeatability represents the standard deviation of these 10 data sets; the average repeatability was $4.48 \mathrm{~nm}$ across $20 \mathrm{~mm}$.

the grating scale geometry adopted by the DiLENS yields an improvement by a factor of 10 while it achieves a lower diffraction variation rate. This lower variation rate can be translated into a better manufacturing tolerance.

A feedback-controlled linear piezoelectric stage was developed as the platform for the DiLENS performance evaluations. To achieve nanometer resolution we used a two-frequency interferometer ${ }^{25}$ as the sensor. To guarantee smooth movement, a Nanomotion SP4-02 ultrasonic motor was driven by use of a sensor to complete the closed-loop control. The laboratory environment was found to have a room temperature of $21.306{ }^{\circ} \mathrm{C}$, with a standard deviation of $0.038^{\circ} \mathrm{C}$. In addition, the average atmospheric pressure was $1.000071 \mathrm{~atm}$, with a standard

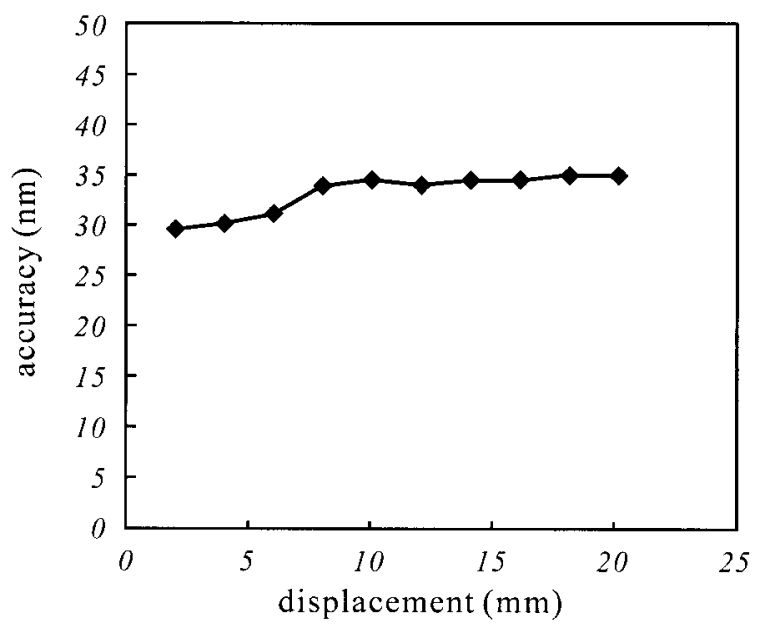

Fig. 12. Experimental results of accuracy tests in which 10 data sets (at 2, 4, 6, 8, 10,12, 14, 16, 18, and $20 \mathrm{~mm}$ ) were obtained. The accuracy represents the root mean square of these 10 data sets; the average accuracy was $33.71 \mathrm{~nm}$ across $20 \mathrm{~mm}$. 


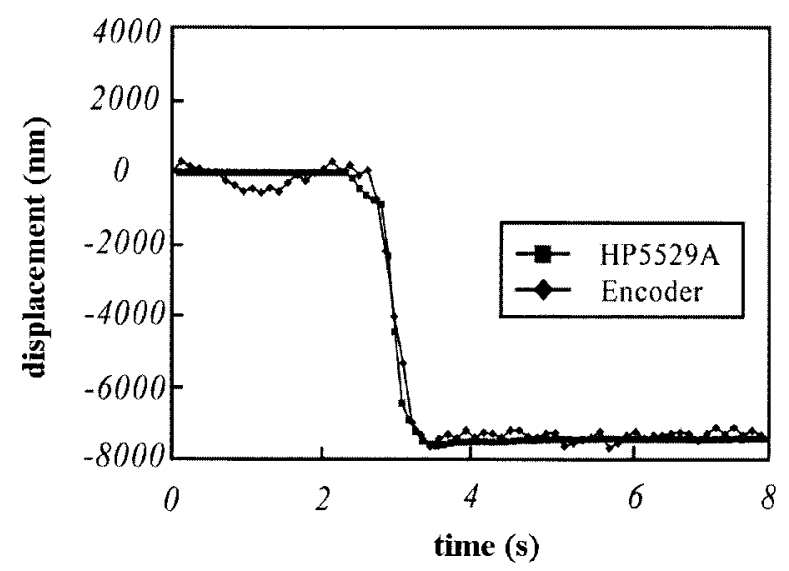

Fig. 13. Comparison of displacement data measured by the DiLENS and by the HP 5529A interferometer.

deviation of $0.0002029 \mathrm{~atm}$. The desk was found to vibrate at $10 \mathrm{~Hz}$, which caused vibration noise. By measuring the data for 10 readings (at $2,4,6,8,10$, $12,14,16,18$, and $20 \mathrm{~mm}$ ) the root-mean-square and standard deviations of these data were obtained. Denoting the standard deviation as the repeatability and the root mean square as the accuracy yields the measured repeatability and accuracy of the DiLENS shown in Figs. 11 and 12, respectively. The results show that the average repeatability achieved was $4.48 \mathrm{~nm}$ and the average accuracy was $33.71 \mathrm{~nm}$. Figure 13 compares the displacement measured by the DiLENS and by the two-frequency interferometer. The discrepancy between these two measurements can be attributed to environmental disturbance and to the degree of accuracy achievable by the two-frequency interferometer, which is $\sim 10 \mathrm{~nm}$.

\section{Conclusions}

A series of newly developed laser encoders that possess high head-to-grating scale tolerance has been designed and constructed. Theoretical analysis has shown that the improved tolerance is due to the built-in folded $1 \times$ telescope that guarantees parallelism between the incident and the returning light beams. The corner-cubelike feature of the folded $1 \times$ telescope was demonstrated by use of both paraxial and ray tracing. A factor-of-6-20 improvement in tolerance compared with that of current state-of-theart diffractive laser encoders was predicted and verified experimentally. In addition, immunity of the DiLENS to differences in diffraction efficiency between the $p$ - and $s$-polarized light beams, which can hinder the performance of traditional diffractive laser encoders, can be seen. The optimal criteria for grating-scale geometry, identified theoretically as well as experimentally, were found to increase further both the signal-to-noise ratio and the gratingscale manufacturing tolerance.

This research was performed at the NanoBioMEMS Laboratory of the Institute of Applied Mechanics, National Taiwan University. This project was partially supported under Ministry of Economic Affairs Department of Industrial Technology Development Program for Academia 91-EC-17-A-05-S10017 and partially supported by the National Science Council of Taiwan under project NSC92-2622-E-002006.

\section{References}

1. N. Bobroff, "Recent advances in displacement measuring interferometry," Meas. Sci. Technol. 4, 907-926 (1993).

2. S. Hosoe, "Highly precise and stable laser displacement measurement interferometer with differential optical passes in practical use," Nanotechnology 4, 81-85 (1993).

3. V. G. Badami and S. R. Petterson, "A frequency domain method for the measurement of non-linearity in heterodyne interferometry," Precis. Eng. 24, 41-49 (2000).

4. V. P. Drachev and S. V. Perminov, "Nonlinearity phase shift without cascaded second-order processes and third-order nonlinearity," Appl. Phys. B 71, 193-196 (2000).

5. D. Lin, H. Jiang, and C. Yin, "Analysis of nonlinearity in a high-resolution grating interferometer," Opt. Laser Technol. 32, 95-99 (2000).

6. W. W. Chiang and C. K. Lee, "Wavefront reconstruction optics for use in disk drive position measurement system," U.S. patent 5,442,172 (15 August 1995).

7. T. Nishimura, M. Tsukiji, S. Ishii, K. Ishizuka, and Y. Kubota, "Optical type encoder," U.S. patent 5,000,542 (19 March 1991).

8. L. Drain, The Laser Doppler Technique (Wiley, New York, 1980).

9. F. Durst, A. Melling, and J. Whitelaw, Principles and Practice of Laser Doppler Anemometry (Academic, New York, 1981).

10. R. Guenther, Modern Optics (Wiley, New York, New York, 1990).

11. W. J. Wu, C. K. Lee, and C. T. Hsieh, "On the signal processing algorithms for Doppler effect based nanometer positioning systems,” Jpn. J. Appl. Phys. Part 1 38, 1725-1729 (1999).

12. A. Cox, A System of Optical Design (Focal Press, New York, 1967).

13. E. Hecht, Optics (Addison-Wesley, New York, 1998).

14. H. Haus, Wave and Fields in Optoelectronics (Prentice-Hall, Englewood Cliffs, N.J., 1984).

15. J. W. Goodman, Introduction to Fourier Optics (McGraw-Hill, New York, 1996).

16. L. Mickens, R. Coblentz, and S. Montepio, LightTools User Guide (Optical Research Associates, Pasadena, Calif., 2000).

17. Canon U.S.A., Model L-104 Laser Linear Encoder (Canon U.S.A., New York).

18. E. G. Leowen and E. Popov, Diffraction Gratings and Applications (Marcel Dekker, New York, 1997).

19. K. G. Masreliez, "Position detection and method of measuring position," U.S. patent 5,104,225 (14 April 1992).

20. E. N. Glytsis and T. K. Gaylord, "Rigorous three-dimensional coupled-wave diffraction analysis of single and cascaded anisotropic grating," J. Opt. Soc. Am. A 4, 2061-2080 (1987).

21. R. Magnusson and T. K. Gaylord, "Equivalence of multiwave coupled-wave theory and modal theory of periodic-media diffraction," J. Opt. Soc. Am. 68, 1777-1779 (1978).

22. M. G. Moharam and T. K. Gaylord, "Formulation for stable and efficient implementation of rigorous coupled-wave analysis of binary gratings," J. Opt. Soc. Am. A 12, 1068-1076 (1995).

23. D. Fluckiger, GSolver User's Manual (Grating Solver Development Company, Allen, Tex., 1991).

24. UDT Instruments, Electronic Autocollimators (UDT Instruments, Baltimore, Md.), http://www.udtinstruments.com.

25. Hewlett-Packard Company, HP 5529A Dynamic Calibrator Measurements Reference Guide (Hewlett-Packard Company, Santa Clara, Calif., 1995). 